\title{
Rhinophyma: Excision and Reconstruction with Local Nasolabial Flap
}

\author{
${ }^{1}$ Yogesh Gajjar, ${ }^{2}$ Rupa Parikh \\ ${ }^{1}$ Assistant Professor, Department of ENT, Surat Municipal Institute of Medical Education and Research (SMIMER), Surat, Gujarat, India \\ ${ }^{2}$ Professor and Head, Department of ENT, Surat Municipal Institute of Medical Education and Research (SMIMER), Surat, Gujarat, India
}

Correspondence: Yogesh Gajjar, Assistant Professor, Department of ENT, S urat Municipal Institute of Medical Education and Research (SMIMER), Surat, Gujarat, India, e-mail: drykggajjar@gmail.com

\section{ABSTRACT}

R hinophyma is a rare disease which causes disfiguring soft tissue hypertrophy of the nose. Various medical and surgical treatment modalities have been proposed and tried with varying degree of success. We report a case where we adopted excision of the rhinophymatous tissue and reconstructed with the local nasolabial flap. The result was cosmetically good enough and the procedure technically simple.

Keywords: R hinophyma, Excision, Nasolabial flap.

\section{INTRODUCTION}

R hinophyma is a descriptive term for a large, bulbous, ruddy appearance of the nose caused by granulomatous infiltration, commonly due to untreated rosacea. ${ }^{1}$ It is a benign dermatologic disease characterized by a slowly progressive enlargement with irregular thickening of the nasal skin and nodular deformation affecting primarily men in their fifth to seventh decade of life. It is assumed to be the end stage of chronic acne rosacea. ${ }^{1} \mathrm{M}$ ain reasons that urge patients to seek hel $p$ are cosmetic and functional impairments. Surgical removal of the hyperplastic tumor mass is the treatment of choice for rhinophyma.

\section{CASE REPORT}

A 73-year-old man presented with complaint of an irregular swelling on the alar region of nose since past 4 to 5 years, which had increased in size over the last six months, on the right side the lesion was bigger than the left side. The patient had no history of alcohol addiction and had no other medical disorder. He had sought medical care due to the unsightly appearance (Fig. 1). The look and its clinical examination pointed towards the diagnosis of rhinophyma. Patient was explained regarding the surgical excision and he wanted the lesion on the right side to be removed as the left sided lesion was small. After basic investigations, the nose was injected with lignocaine containing adrenaline to reduce bleeding, and the lesion was excised completely on the right side up to the underlined cartilage and the defect was reconstructed by local nasolabial flap, and the postoperative cosmetic result was very good after a week (Fig. 2) and after 3 months (Fig. 3).

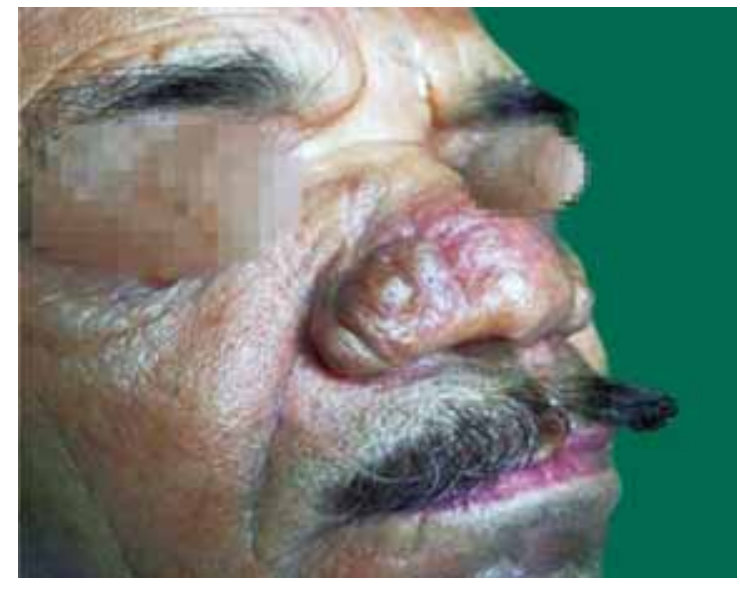

Fig. 1: Preoperative photograph

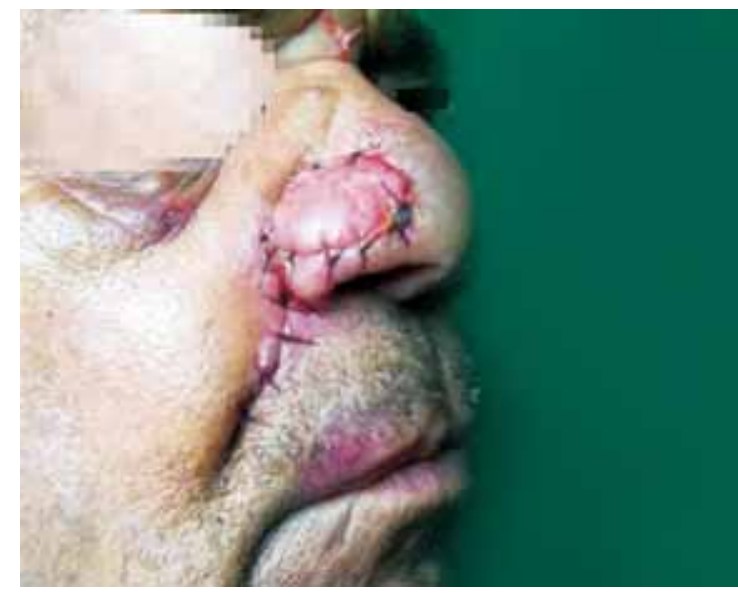

Fig. 2: Postoperative photograph after 7 days

\section{DISCUSSION}

Identifying the symptoms of rhinophyma is essential to a proper diagnosis and treatment. The symptoms are relatively 


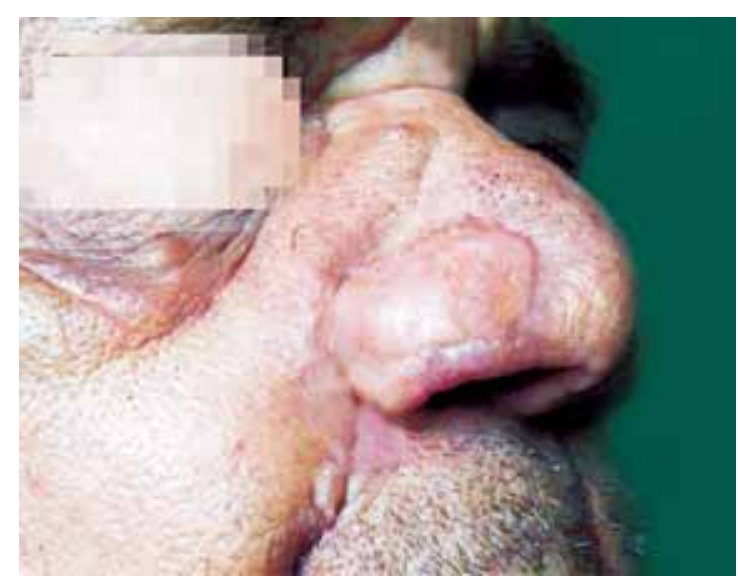

Fig. 3: Postoperative photograph after 3 months

unique to this condition and are apparent to patient and surgeon. Symptoms include an inflamed, bulb shaped nose with thick skin. The skin of the nose may appear to be red or reddish, or yellow and waxy. Rhinophyma can be diagnosed with a simple visual examination. Due to the unique appearance of this condition, there is usually no need for any additional tests. Etiological factors, such as alcohol, hot and spicy foods and exposure to climatic extremes, are not consistent findings and are not believed to be involved. ${ }^{2}$

The microscopic progress is from initial hyperemia to permanent dilatation of arterioles, venules and capillaries, along with proliferation of new blood vessels. There is transudation of edema fluid resulting in fibrosis and obstruction of the sebaceous glands. These dilate and hypertrophy, producing sebum-filled cysts, which give the characteristic nodular appearance to the growth and its accompanying unpleasant odor.

A wide range of treatments have been advocated, both surgical and nonsurgical. While the nonsurgical, i.e. medical treatment ${ }^{3}$ had a place in early cases, acne rosacea may respond to local medical or dermatologic treatment and prevent progression to rhinophyma. Medical treatment classically has been limited to the avoidance of stimulation factors, appropriate cleanliness, and treatment of secondary infection and inflammation with antibiotics and steroids. Systemic therapy includes oral antibiotics, such as flagyl (250 mg/day $\times 10$ days) or tetracycline $(250 \mathrm{mg}$ QID or $500 \mathrm{mg} \mathrm{BID}$ ) can be used in more long-term management but is often less effective. Isotretinoin (13-cis-retinoic acid "acutane") in doses of 0.2 to $1.0 \mathrm{mg} / \mathrm{kg}$ for 10 to 16 weeks has been shown to provide up to a $90 \%$ reduction in the volume of sebaceous glands. It is generally accepted that surgery is the only treatment likely to benefit established rhinophyma. Surgical treatment of rhinophyma includes electrosurgery, ${ }^{4}$ laser treatment, ${ }^{5}$ dermabrasion. ${ }^{6}$ Electrosurgery, ${ }^{4}$ i.e. excision using high-frequency electrosurgery with a wire loop provides a quick, efficient, bloodless operative field with a good cosmetic result and pain-free postoperative recovery. U se of laser either $\mathrm{CO}_{2}$ or ND:Y A G provides very accurate tissue ablation and allows the sculpturing of the hypertrophied areas, offering good cosmetic results, with a very short healing period. ${ }^{5}$ Dermabrasion ${ }^{6}$ describes the mechanical removal of the epidermis or both the epidermis and dermis. It can be used to improve the appearance of acne scars as well as prominent/deep wrinkles. It is also utilized to sculpt the nose in cases of rhinophyma.

Complete surgical removal of the lesion and subsequent reconstruction by local flaps ${ }^{7}$ is a good option for better postoperative cosmetic results and this case report is based on this treatment modality. N asolabial flaps are random flaps vascularized by subdermal plexus, which run along the long axis of the flap. ${ }^{8}$ Superiorly based nasolabial transposition flaps are best suited for al ar reconstruction in el derly patients and patients who are not demanding about the esthetic outcome or those who do not wish a staged reconstruction. ${ }^{8}$ Cameron, L atham and Dowling illustrate the use of nasolabial transposition flaps in nasal surgery. ${ }^{9}$

Recently, the microdebrider excision along with the use of the hemostatic agent application has also been reported. ${ }^{10}$

\section{CONCLUSION}

This case reveals that surgical excision along with reconstruction by local flap is a good option for improved postoperative cosmetic results for patients suffering from rhinophyma.

\section{REFERENCES}

1. Cohen AF, Tiemstra JD. Diagnosis and treatment of rosacea. J A m B oard Fam Pract 2002;15(3):214-17.

2. K evin B erman. A tlanta center for dermatologic disease, A tlanta, GA. Review provided by VeriM ed Healthcare Network 26 October 2006.

3. Pastorek NJ. The management of rhinophyma. Otolaryngol Clin N A m 1972;5:639-66.

4. Clark DP, HankeCW . Electrosurgical treatment of rhinophyma. J Am A cad Dermatol M ay 1990;22(5 Pt 1):831-37.

5. L aser A rie O renstein, J osef H aik, J eremy Tamir, Eyal Winkler, J acob Frand, Isaac Zilinsky, H aim K aplan. Treatment of rhinophyma with Er:YAG. Lasers in Surgery and Medicine 29(3):230-35.

6. B aker TM . D ermabrasion as a complement to aesthetic surgery. Clin Plast Surg 1998 J an;25(1):81-88.

7. Hynes B , B oyd JB. The nasiolabial flap: A xial or random? A rch Otolaryngol Head Neck Surg 1988;114:1389-91.

8. $M C L$ aren $L R$. N asolabial flap repair for alar margin defects. $\mathrm{Br}$ J Plast Surg 1963;16:234-38.

9. Cameron RR, Latham WD, Dowling JA. Reconstructions of the nose and upper lip with nasolabial flaps. Plast Reconstr Surg 1973;52:145.

10. K aushik V, Tahery J, M alik TH, J ones PH. M icrodebrider and Floseal. The Journal of Laryngology and Otology $2003 \mathrm{Jul}$; 117(7):551-52. 